\title{
Incidencia de la motivación en la producción científica institucional
}

\section{Impact of motivation in institutional scientific production}

\author{
Marcelo Bastidas Jiménez* \\ mbastidas@ups.edu.ec \\ Rosa Benites Medina** \\ rbenites@ups.edu.ec
}

\begin{abstract}
Resumen
Entendiendo la motivación como el principal factor para el accionar de un individuo, este estudio abordó los factores motivacionales involucrados en la decisión de un docente hacia la producción científica y la conformación de Grupos de Investigación en la carrera de Administración de Empresas de la Universidad Politécnica Salesiana en Guayaquil. El trabajo expuesto a través del presente artículo comprendió una investigación de carácter cuantitativa y una cualitativa. Los resultados mostraron que si bien es cierto hay desconocimiento de los reglamentos y procesos asociados con la producción científica, existe una alta motivación al respecto. Los factores de motivación son variados, pero casi un $60 \%$ de los docentes no se enfocaron en asuntos monetarios, lo cual fue alentador.
\end{abstract}

\section{Palabras clave}

Administración, docentes, motivación, producción científica.

Ecuatoriano. Universidad Politécnica Salesiana- Guayaquil. Máster en Administración en Empresas. Docente.

* Ecuatoriana. Universidad Politécnica Salesiana- Guayaquil. Máster en Administración de Empresas y Comercio Electrónico. Docente. 


\begin{abstract}
Understanding motivation as the main factor for the actions of an individual, this study addressed the motivational factors involved in the decision of a teacher to the scientific production and the formation of Research Groups in the career of Business Administration from the Salesian Polytechnic University in Guayaquil. The work exposed through this article included a quantitative and qualitative research. The results showed that while it is true there is a lack of regulations and processes associated with scientific production, there is a high motivation in this regard. The motivating factors are varied, but almost $60 \%$ of teachers did not focus on monetary affairs, which was encouraging.
\end{abstract}

\title{
Keywords
}

Administration, teachers, motivation, scientific production.

Forma sugerida de citar: Bastidas Jiménez, Marcelo, \& Benites Medina, Rosa (2016). Incidencia de la motivación en la producción científica institucional. Revista Retos, 11(1), pp.65-87.

\section{Introducción}

La Ley de Educación Superior del Ecuador, LOES, pone de manifiesto como uno de los fines de la educación superior a la producción científica y a las transferencias e innovaciones tecnológicas como un aporte al desarrollo del pensamiento universal. También las Instituciones de Educación Superior deben asignar recursos de sus propios presupuestos para la publicación de las investigaciones en revistas indexadas de sus investigadores (Presidencia de la República, 2010).

Tal es la importancia de la producción científica, que dentro del nuevo modelo genérico de evaluación del entorno de aprendizaje de carreras presenciales y semipresenciales de las Universidades y Escue- las Politécnicas del Ecuador, ligado al criterio academia, se encuentra como tercer subcriterio la producción académica, la cual envuelve una serie de cuatro indicadores, que son: Producción académico científica, producción regional, libros o capítulos de libros y ponencias (Consejo de Evaluación, Acreditación y Aseguramiento de la calidad de la Educación Superior, 2015).

Las publicaciones son el componente clave de toda actividad científica (Peralta, Solis \& Peralta, 2010), y siendo la investigación uno de los pilares fundamentales de la educación superior en la actualidad, junto con la docencia y la vinculación. Entonces como la producción científica se constituye en una pieza clave dentro de la calidad de la Educación 
Superior (Álvarez \& Juncosa, 2014), puesto que es una forma en la que las instituciones educativas ejercen una función de difundir los conocimientos generados dentro de su seno. Esta producción que se genera al interior de las Instituciones de Educación Superior, no solo es un aporte para la ciencia, sino que al mediano y largo plazo proporciona pautas para la transformación de su entorno social (Gómez, Siles \& Tejedor, 2012).

Esto deja una reflexión muy relevante en el sentido de la importancia de la producción científica en el beneficio del bien común (Abadal, 2013), debido a que el acercamiento de las universidades hacia su entorno a través de la llamada vinculación con la colectividad aumenta la producción científica de su claustro docente a su vez beneficia a la colectividad a través de la creación de conocimiento significativo, que puede por un lado resolver problemas actuales del entorno social y por otro lado generar nuevas oportunidades para las empresas y para la sociedad en general.

\section{Producción científica ecuatoriana}

Ecuador viene experimentando un crecimiento promedio anual del $10.8 \%$ en la cantidad de artículos publicados revistas indexadas en en Scopus en el decenio del 2003 al 2012. Dentro del mismo período experimenta un crecimiento promedio anual del $9.4 \%$ en la cantidad de artículos publicados en ISI Web of Knowledge (Bruque, 2014).

Tabla 1. Producción científica ecuatoriana en el decenio 2003 - 2012

\begin{tabular}{|c|c|c|}
\hline Año & No. Artículos Scopus & $\begin{array}{c}\text { No. Artículos ISI Web of } \\
\text { Knowledge }\end{array}$ \\
\hline 2003 & 203 & 220 \\
\hline 2004 & 207 & 208 \\
\hline 2005 & 281 & 269 \\
\hline 2006 & 296 & 289 \\
\hline 2007 & 333 & 374 \\
\hline 2008 & 394 & 380 \\
\hline 2009 & 475 & 461 \\
\hline 2010 & 431 & 392 \\
\hline 2011 & 463 & 439 \\
\hline 2012 & 566 & 541 \\
\hline $2003-2012$ & 3649 & 3573 \\
\hline Crecimiento promedio anual & $10,8 \%$ & $9,4 \%$ \\
\hline
\end{tabular}


A pesar del crecimiento Ecuador sigue representando un país con muy poca participación científica en el contexto latinoamericano. Prueba de ello es que se puede ver al país en una posición intermedia dentro de los países de la región, por encima de países como Panamá, Bolivia y Guatemala, pero por debajo de Perú, Colombia, Venezuela y Chile.

Tabla 2. Producción científica en el decenio 2003-2012 de algunos países latinoamericanos y del Caribe

\begin{tabular}{|c|c|c|}
\hline País & No. Artículos Scopus & $\begin{array}{l}\text { No. Artículos ISI Web of } \\
\text { Knowledge }\end{array}$ \\
\hline Chile & 54754 & 61988 \\
\hline Colombia & 31188 & 29666 \\
\hline Venezuela & 18884 & 18043 \\
\hline Cuba & 16937 & 12172 \\
\hline Puerto Rico & 8096 & 11184 \\
\hline Perú & 7544 & 9183 \\
\hline Uruguay & 7281 & 8945 \\
\hline Costa Rica & 4755 & 5196 \\
\hline Ecuador & 3651 & 3935 \\
\hline Panamá & 2890 & 4602 \\
\hline Bolivia & 1945 & 2366 \\
\hline Guatemala & 1143 & 1286 \\
\hline Paraguay & 752 & 1510 \\
\hline Nicaragua & 771 & 750 \\
\hline El Salvador & 671 & 464 \\
\hline Honduras & 496 & 548 \\
\hline Haití & 372 & 423 \\
\hline
\end{tabular}

Fuente: SENESCYT, 2013 
Entre los años 2005 y 2009 Miguel Sandra realizó un trabajo sobre la presencia de revistas de la región latinoamericana y del Caribe en índices como Redalyc, Scielo y Scopus, como se observa en la tabla 3 el país con mayor cantidad de títulos fue Brasil, seguido de México, Colombia, Chile, Argentina y Venezuela. Entre esos seis países se cuenta el $89.7 \%$ de todas las revistas visibilizadas en las tres fuentes. Ecuador por su parte contaba con 5 títulos diferentes, de los cuales ninguno se visibiliza en Scielo (Sandra, 2011).

\section{Tabla 3. Presencia de revistas de países de Latinoamérica y El Caribe en Scielo, Redalyc y Scopus}

\begin{tabular}{|c|c|c|c|c|c|c|c|c|}
\hline \multirow{2}{*}{ Países } & \multicolumn{2}{|c|}{ Scielo } & \multicolumn{2}{|c|}{ Redalyc } & \multicolumn{2}{|c|}{ Scopus } & \multirow{2}{*}{$\begin{array}{l}\text { Total de } \\
\text { revistas } \\
\text { diferentes }\end{array}$} & \multirow{2}{*}{$\%$} \\
\hline & $\begin{array}{c}\# \\
\text { revistas }\end{array}$ & $\%$ & $\begin{array}{c}\# \\
\text { revistas }\end{array}$ & $\%$ & $\begin{array}{c}\# \\
\text { revistas }\end{array}$ & $\%$ & & \\
\hline Argentina & 74 & 10,3 & 41 & 6,4 & 41 & 7,8 & 110 & 8,8 \\
\hline Bolivia & 6 & 0,8 & 0 & 0 & 1 & 0,2 & 7 & 0,6 \\
\hline Brasil & 253 & 35,2 & 117 & 117 & 234 & 44,7 & 384 & 30,8 \\
\hline Chile & 93 & 12,9 & 66 & 66 & 69 & 13,2 & 120 & 9,6 \\
\hline Colombia & 107 & 14,9 & 138 & 138 & 37 & 7,1 & 181 & 14,5 \\
\hline Costa Rica & 11 & 1,5 & 16 & 16 & 1 & 0,2 & 24 & 1,9 \\
\hline Cuba & 36 & 5 & 19 & 19 & 21 & 4 & 51 & 4,1 \\
\hline Ecuador & 0 & 0 & 3 & 3 & 2 & 0,4 & 5 & 0,4 \\
\hline Jamaica & 0 & 0 & 0 & 0 & 2 & 0,4 & 2 & 0,2 \\
\hline México & 77 & 10,7 & 169 & 169 & 70 & 13,4 & 215 & 17,3 \\
\hline Paraguay & 3 & 0,4 & 0 & 0 & 0 & 0 & 3 & 0,2 \\
\hline Perú & 13 & 1,8 & 11 & 11 & 2 & 0,4 & 20 & 1,6 \\
\hline Puerto Rico & 0 & 0 & 4 & 4 & 4 & 0,8 & 8 & 0,6 \\
\hline $\begin{array}{l}\text { Rep. Domini- } \\
\text { cana }\end{array}$ & 0 & 0 & 1 & 1 & 0 & 0 & 1 & 0,1 \\
\hline Uruguay & 5 & 0,7 & 1 & 1 & 0 & 0 & 6 & 0,5 \\
\hline Venezuela & 41 & 5,7 & 56 & 56 & 39 & 7,5 & 109 & 8,7 \\
\hline
\end{tabular}

Fuente: (Sandra, 2011) 
Motivación y producción científica

La motivación no es otra cosa que el conjunto de deseos que lanzan a la acción al individuo (Marina, 2011). De allí que su entendimiento se considera tan importante en la vida, puesto que todas las personas en algún momento desean motivar a otros para que ejecuten acciones específicas e incluso buscar la forma de automotivarse.

Es claro que la motivación en los ambientes laborales es el resultados de una serie de factores, tanto individuales como grupales, intrínsecos como extrínsecos (Gan \& Triginé, 2012). Entre estos factores se pueden citar: la situación personal y/o familiar del individuo, sus metas profesionales, el ambiente laboral, la comunicación entre pares y hacia los directivos, la forma en la que se abordan los conflictos, entre otros. La motivación puede clasificarse por su sentido, por su génesis y por su objeto (Peña, Macías \& Morales, 2011). Nadie en un sentido estricto, puede obligar a otro a motivarse, debido a que la motivación es interna a la persona y radica en el cerebro del individuo, pero si existen motivadores, los cuales pueden estimular la acción del individuo (Fischman, 2014).

Los ambientes universitarios hoy en día no distan en gran medida de los ambientes laborales, puesto que un docente tiene una jornada laboral y debe cumplir una serie de obligaciones en la universidad. Dichas obliga- ciones se relacionan en gran medida con la cátedra, pero también involucran otras actividades como son las tutorías académicas, revisiones de trabajos de culminación de carrera, actividades de vinculación con la sociedad y tareas relacionadas con la investigación y producción científica.

Es menester pensar que la producción científica como cualquier otra actividad es susceptible de estar alineada a los gustos y necesidades de los docentes dentro de una institución de Educación Superior (Pirela \& Prieto, 2006), de allí que para algunos docentes ésta puede ser una actividad que despierte su motivación, mientras que para otros genere incomodidad. Lo que sí es cierto es que la labor de investigación y la transformación de conocimientos en una producción científica requieren del docente ciertas competencias (Bozu \& Canto, 2009) que en muchos de los casos no son innatas a la persona, por lo cual pueden potenciarse con la formación adecuada.

Según lo expuesto se plantea la siguiente pregunta de investigación: ¿Es necesario que en las universidades se exploren los factores que generan motivación de los docentes hacia la producción científica?, de este modo se puede incentivar al cuerpo docente a mejorar la cantidad y calidad de dicha producción entendiendo que esto incidirá positivamente en los procesos de evaluación y acreditación en el Ecuador. 


\section{Metodología}

El trabajo de campo involucró la realización de dos investigaciones, una cuantitativa y una cualitativa. La primera fue dirigida a los docentes de la carrera de Administración de Empresas de la Universidad Politécnica Salesiana de la ciudad de Guayaquil; la segunda por su parte fue direccionada a dos personas involucradas dentro del proceso de producción científica. Las investigaciones fueron realizadas entre marzo y mayo del año 2015.

\section{Estudio cuantitativo}

Población y muestra

$$
\begin{aligned}
& n=\frac{Z^{2} P Q N}{e^{2}(N-1)+Z^{2} P Q} \\
& n=\frac{(1,96)^{2} \times 0,5 \times 0,5 \times 85}{(0,05)^{2} \times 84+(1,96)^{2} \times 0,5 \times 0,5} \\
& n \approx 70
\end{aligned}
$$

La población objetivo para la parte cuantitativa estuvo constituida por todos los docentes de la carrera de Administración de Empresas de la Universidad Politécnica Salesiana. Esta población se ha cuantificado en ochenta y cinco individuos.

La muestra fue calculada considerando un nivel de confianza del 95\%, un error máximo admisible del $5 \%$ y una proporción poblacional ajustada de 0,5. El resultado del cálculo puede apreciarse a continuación:

El tamaño de muestra calculado fue de 70 docentes. El método de levantamiento de información empleado fue la entrevista personal, el instrumento utilizado para tal efecto fue un cuestionario estructurado, el cual fue validado por expertos de la misma universidad.

\section{Estudio cualitativo}

Para la parte cualitativa, la población de interés son todos aquellos directivos involucrados directa o indirectamente con las políticas de promoción de la investigación y producción científica de la sede Guayaquil. Convenientemente se direccionaron las entrevistas a dos personas en particular: Ing. Fabián Villacrés Beltrán, Director de la Carrera de Administración de Empresas, Dra. Raquel Ayala Carabajo, Coordinadora de Investigación de Sede.

\section{Resultados}

Encuestas a docentes de la Carrera de Administración de Empresas en Guayaquil

La muestra tomada para el estudio se caracterizó en términos de tres variables: Sexo, Dedicación y años en la docencia. Respecto del sexo, la muestra estuvo conformada por un $62.5 \%$ de hombres y un $37.5 \%$ de mujeres. Gráfico 1. 


\section{Gráfico 1. Composición de la muestra por sexo}

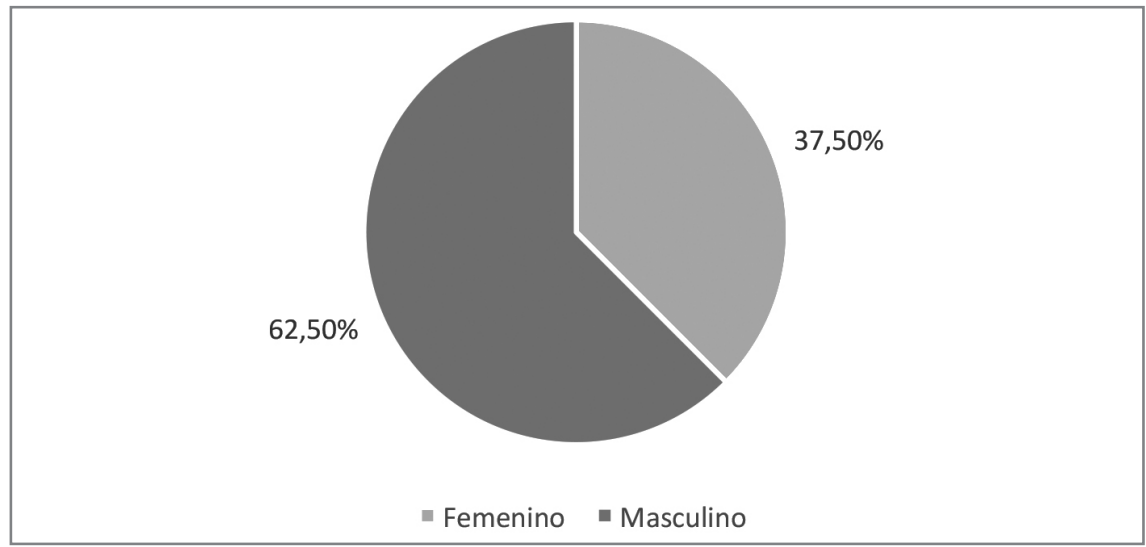

Fuente: Encuesta a docentes de la carrera de Administración de Empresas, UPS - Guayaquil

Sobre la dedicación, el $83.8 \%$ de con dedicación a tiempo completo y docentes encuestados fueron aquellos el 16.3\% medio tiempo. Gráfico 2.

\section{Gráfico 2. Composición de la muestra por dedicación del docente}

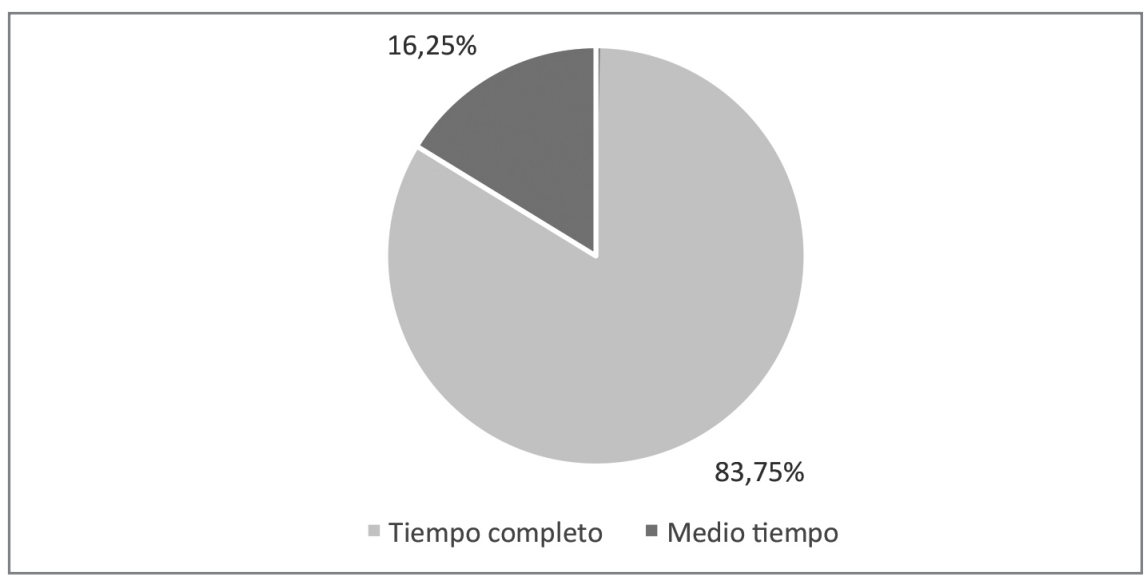

Fuente: Encuesta a docentes de la carrera de Administración de Empresas, UPS - Guayaquil

Finalmente en cuanto a los años de docencia, el $31.3 \%$ de los encuestados tenían menos de dos años en la docencia, un 30\% tenían entre dos y cinco años, un $12.5 \%$ contaba entre seis y diez años y, el 26.3\% de ellos indicó contar con más de diez años en el ejercicio de la actividad docente. Gráfico 3. 
Gráfico 3. Composición de la muestra por años de docencia

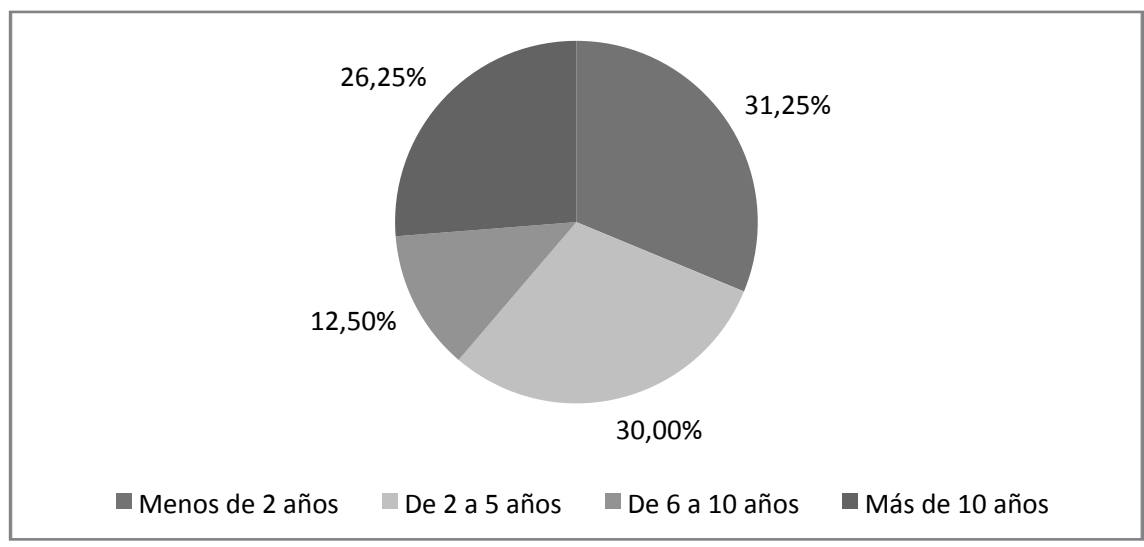

Fuente: Encuesta a docentes de la carrera de Administración de Empresas, UPS - Guayaquil

En las dos preguntas iniciales de grupos de investigación en la Univerla encuesta se indagó sobre el conoci- sidad Politécnica Salesiana, El 53.8\% miento de los profesores sobre los re- de los docentes encuestados indicó glamentos referentes a las publicacio- conocer el reglamento concerniente a nes científicas y a la conformación de las publicaciones científicas. Gráfico 4

\section{Gráfico 4. Conocimiento de la reglamentación sobre publicaciones científicas}

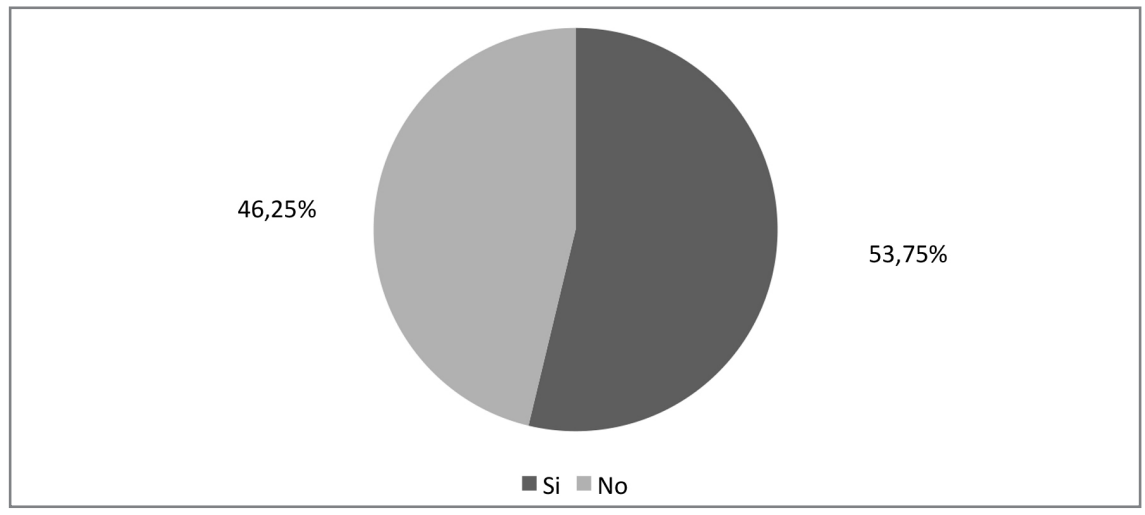

Fuente: Encuesta a docentes de la carrera de Administración de Empresas, UPS - Guayaquil

Así mismo un 35\% afirmó conocer el reglamento para la conformación de grupos de investigación. Gráfico 5. 
Gráfico 5. Conocimiento de la reglamentación sobre conformación de grupos de investigación

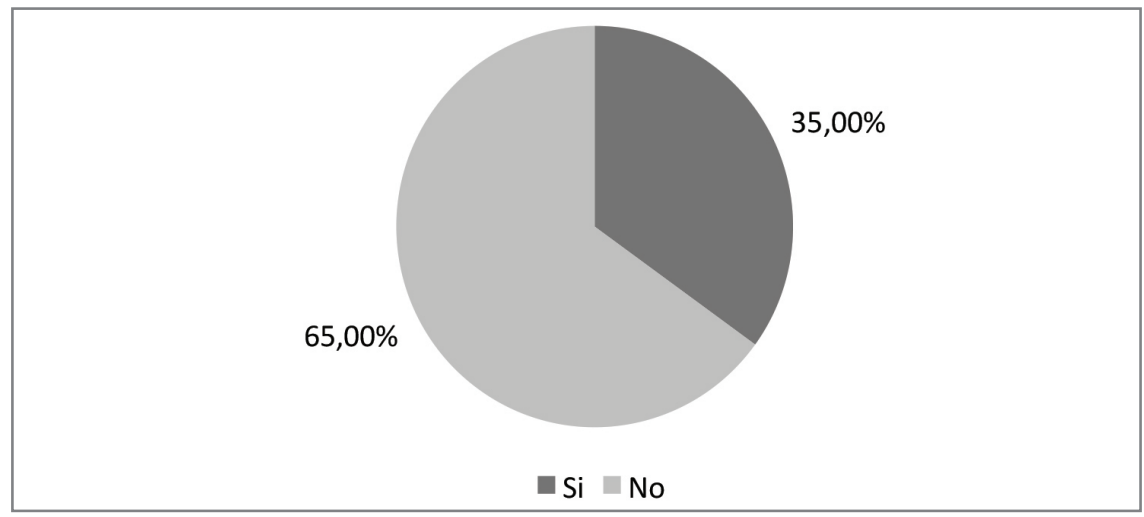

Fuente: Encuesta a docentes de la carrera de Administración de Empresas, UPS - Guayaquil.

En la misma línea se preguntó los docentes contestaron que no persobre la conformación de grupos de tenecen a grupo alguno. Gráfico 6. investigación a lo cual el $87.5 \%$ de

\section{Gráfico 6. Conformación de grupos de investigación}

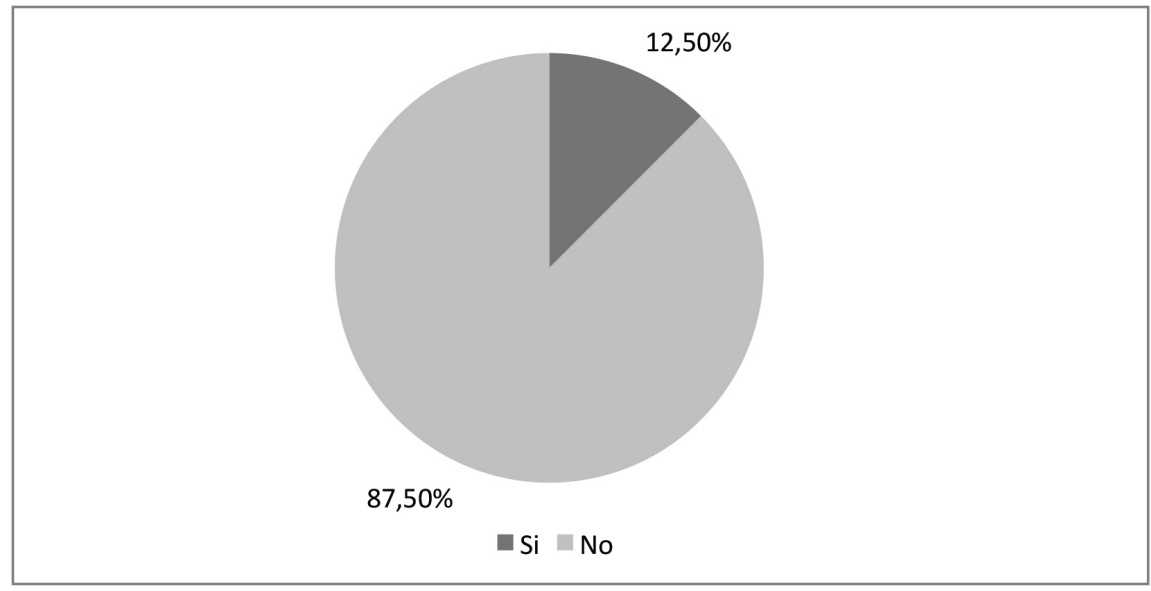

Fuente: Encuesta a docentes de la carrera de Administración de Empresas, UPS - Guayaquil

Sobre el conocimiento de herramientas básicas para la producción científica, se les pidió a los docentes evaluar sus conocimientos en investigación científica, redacción científica, uso de tecnología, lectura en 
inglés y uso de herramientas estadísticas. En investigación científica el $25.1 \%$ considera tener poco o deficiente conocimiento, el $35 \%$ asegura tener un conocimiento moderado y el $40 \%$ considera que tiene bastante o suficiente conocimiento. Gráfico 7.

\section{Gráfico 7. Conocimientos de investigación científica}

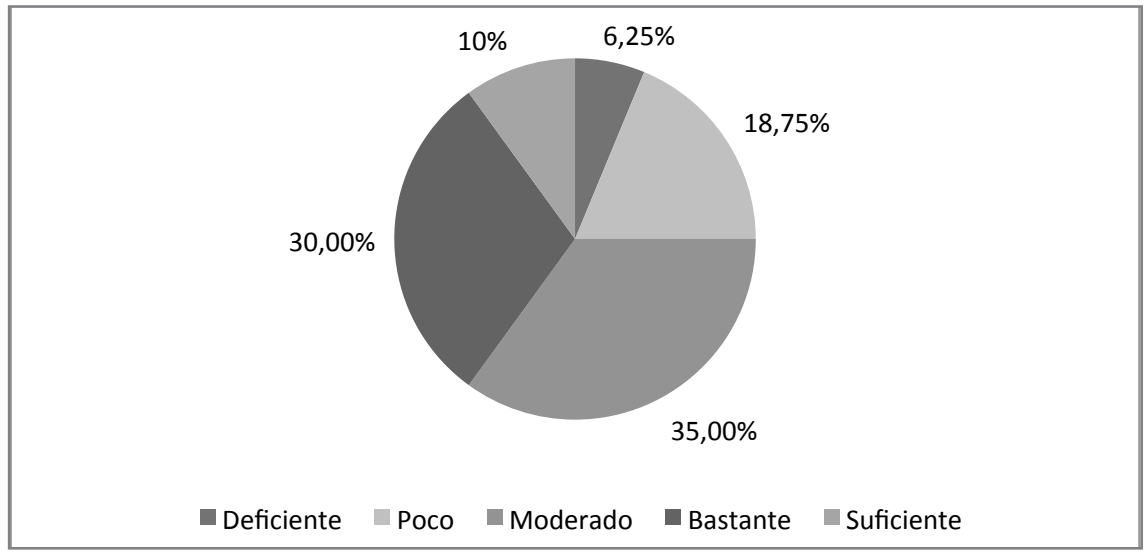

Fuente: Encuesta a docentes de la carrera de Administración de Empresas, UPS - Guayaquil

En redacción científica, el nocimiento es moderado y el $38.8 \%$ $22.5 \%$ considera tener pocos conoci- de ellos indica que es bastante o sufimientos, el 38.8\% afirma que su co- ciente. Gráfico 8.

\section{Gráfico 8. Conocimientos de redacción científica}

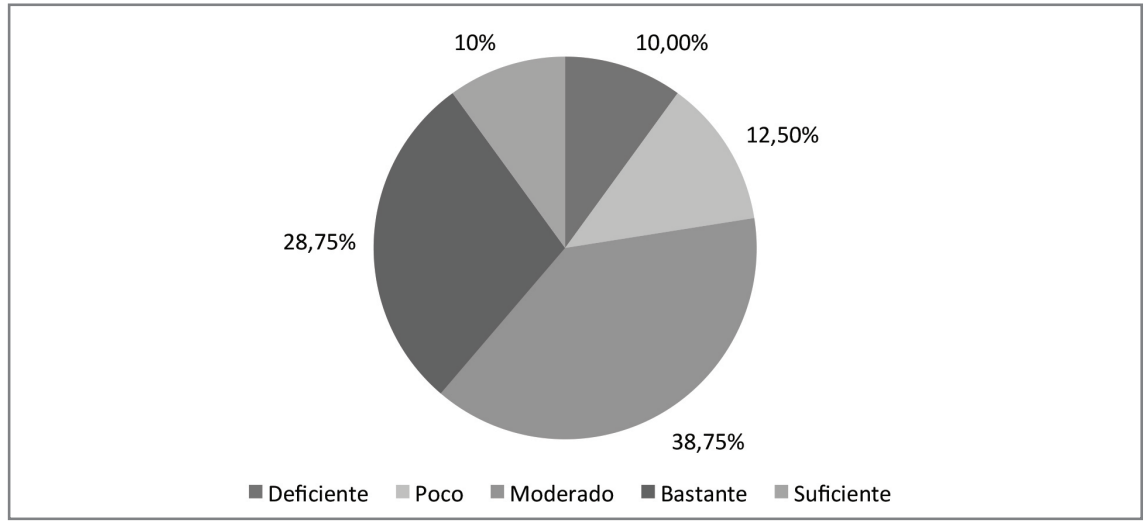

Fuente: Encuesta a docentes de la carrera de Administración de Empresas, UPS - Guayaquil 
En relación al uso de la tecnolo- $17.5 \%$ afirma tener un conocimiento gía, solo un 5\% de los encuestados con- moderado y el $77.5 \%$ considera tener sidera tener poco conocimiento, un bastante o suficiente conocimiento.

\section{Gráfico 9. Conocimientos de uso de la tecnología}

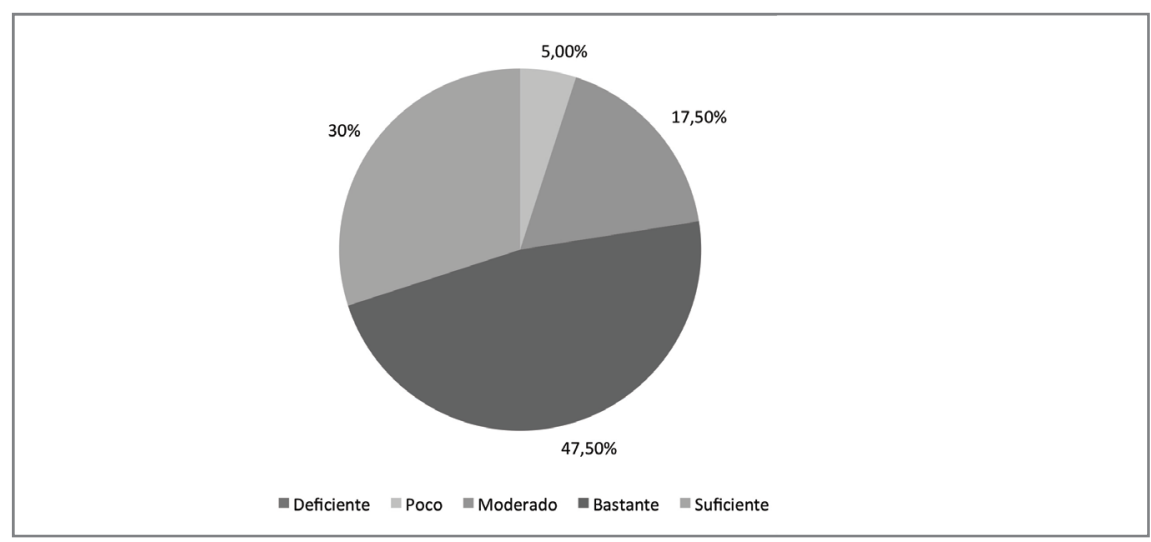

Fuente: Encuesta a docentes de la carrera de Administración de Empresas, UPS - Guayaquil

Sobre la lectura en inglés, el mó tener un conocimiento moderado $28.8 \%$ de los docentes considera te- y un $38.8 \%$ considera tener altos coner muchas falencias, el 32.5\% afir- nocimientos. Gráfico 10.

\section{Gráfico 10. Conocimientos de lectura en inglés}

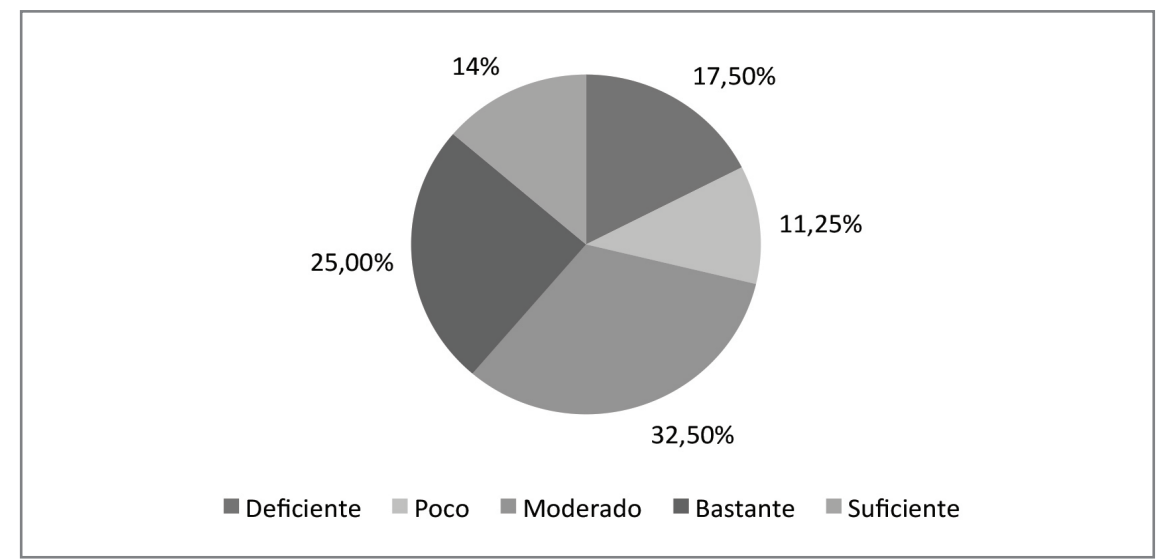

Fuente: Encuesta a docentes de la carrera de Administración de Empresas, UPS - Guayaquil 
Finalmente en relación a las herramientas estadísticas, el $30.1 \%$ indicó tener insuficientes conocimientos, el $32.5 \%$ se manifestó con conocimiento moderado y el $47.5 \%$ de ellos considera tener un conocimiento suficiente. Gráfico 11.

\section{Gráfico 11. Conocimientos de herramientas estadísticas}

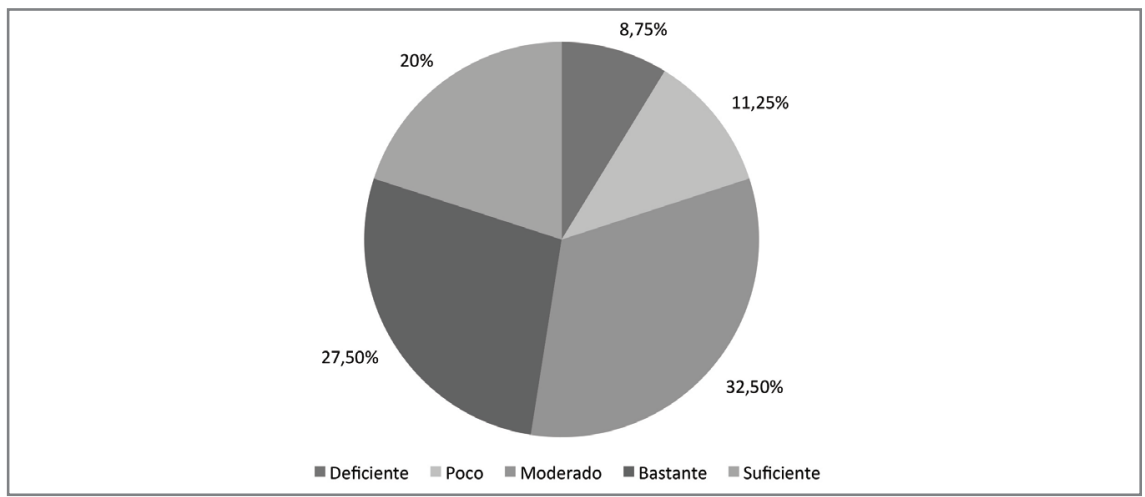

Fuente: Encuesta a docentes de la carrera de Administración de Empresas, UPS - Guayaquil

Posteriormente se hicieron pre- el 46.3\% indica estar medianamente guntas sobre la motivación frente motivado y un 10\% de ellos estaría a la producción científica, a lo cual poco o nada motivado. Gráfico 12. 43.8\% manifestó estar muy motivado,

\section{Gráfico 12. Motivación frente a la producción científica}

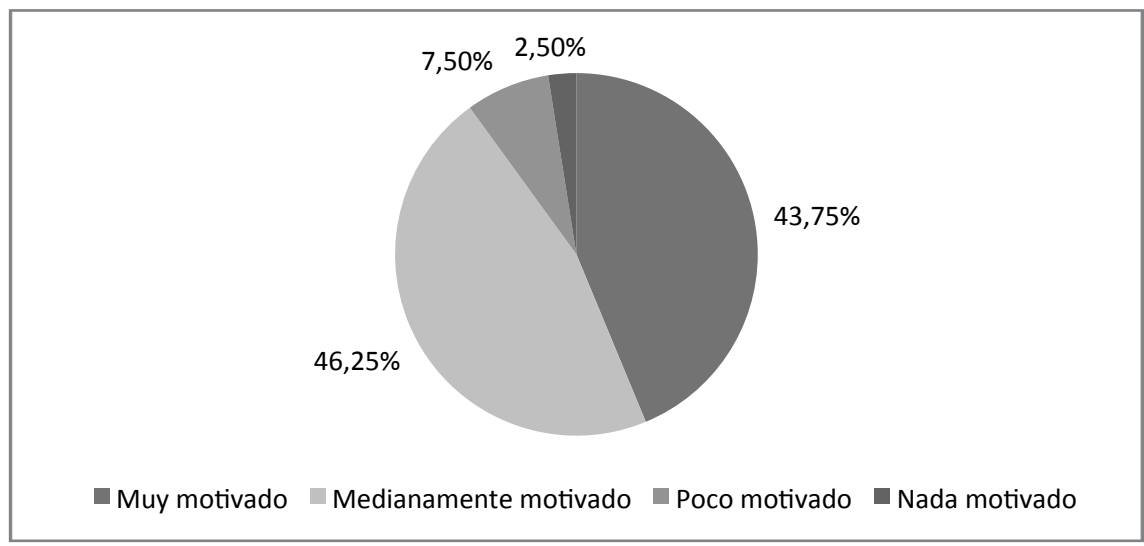

Fuente: Encuesta a docentes de la carrera de Administración de Empresas, UPS - Guayaquil 
Complementariamente se preguntó sobre el factor que haría que dicha motivación aumente; el 21.8\% de las respuestas se centraron en los incentivos monetarios, un $20.4 \%$ en el prestigio, el $19.7 \%$ de las respuestas se enfocó en las mejoras de escalafón docente, el 15\% se concentró en el conocimiento adquirido y compartido, el $13.6 \%$ enfocó la capacitación asociada con los procesos de producción científica, el resto de respuestas se enfocaron en temas de estabilidad laboral y tiempo.

Tabla 4. Factor que aumentaría la motivación

\begin{tabular}{|c|c|c|c|c|}
\hline & & \multicolumn{2}{|c|}{ Respuestas } & \multirow{2}{*}{ Porcentaje de casos } \\
\hline & & No & Porcentaje & \\
\hline \multirow{7}{*}{ Motivación ${ }^{a}$} & Incentivos monetarios & 32 & $21,8 \%$ & $40,0 \%$ \\
\hline & Prestigio & 30 & $20,4 \%$ & $37,5 \%$ \\
\hline & Conocimientos & 22 & $15,0 \%$ & $27,5 \%$ \\
\hline & Mejorar escalafón & 29 & $19,7 \%$ & $36,2 \%$ \\
\hline & Estabilidad laboral & 10 & $6,8 \%$ & $12,5 \%$ \\
\hline & Cpacitación & 20 & $13,6 \%$ & $25,0 \%$ \\
\hline & Tiempo & 4 & $2,7 \%$ & $5,0 \%$ \\
\hline Total & & 147 & $100,0 \%$ & $183,7 \%$ \\
\hline
\end{tabular}

Fuente: Encuesta a docentes de la carrera de Administración de Empresas, UPS - Guayaquil

Luego de indagar sobre los factores motivacionales, se realizaron preguntas sobre la producción científica actual de los docentes de la carrera. En primer lugar se preguntó sobre si el docente ha publicado artículos en revistas no indexadas, a lo que el 25\% contestó afirmativamente y un $75 \%$ de ellos respondió negativamente. Gráfico 13. 
Gráfico 13. Artículos publicados en revistas no indexadas

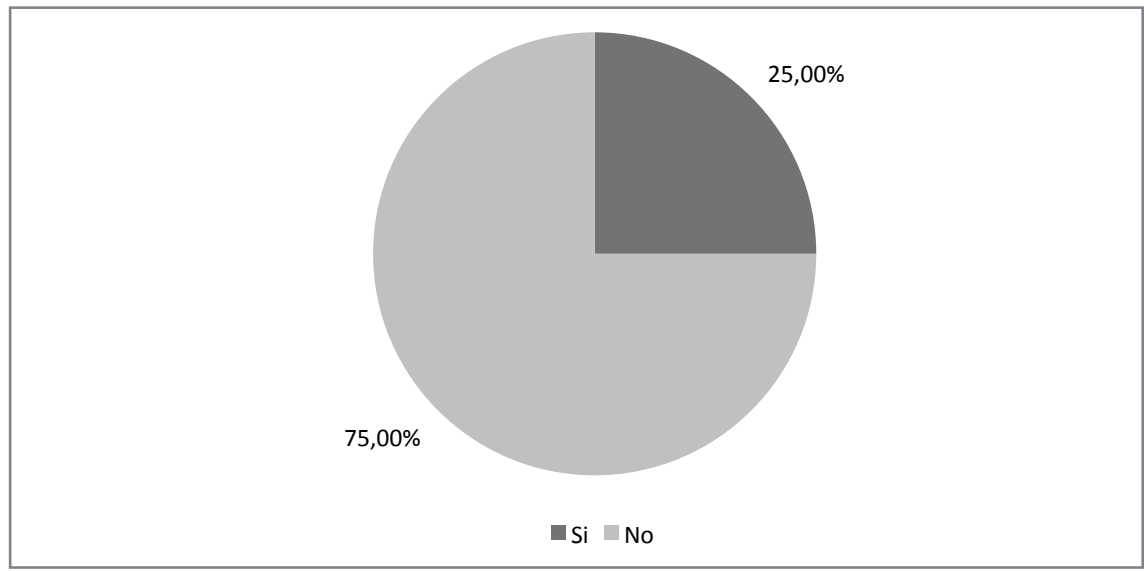

Fuente: Encuesta a docentes de la carrera de Administración de Empresas, UPS - Guayaquil

Sobre la publicación de artícu- tras que el $88.75 \%$ de ellos nunca los en revistas indexadas, un $25 \%$ de ha publicado en revistas indexadas. los docentes ya lo han hecho, mien- Gráfica 14 .

\section{Gráfico 14. Artículos publicados en revistas indexadas}

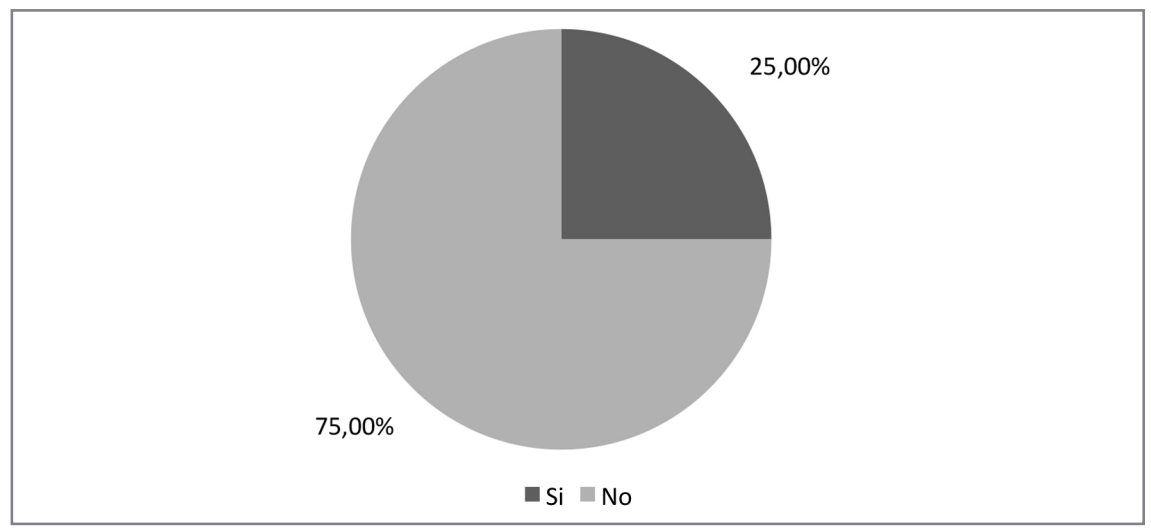

Fuente: Encuesta a docentes de la carrera de Administración de Empresas, UPS - Guayaquil

El $27.5 \%$ de los docentes de la otro $72.5 \%$ de ellos no lo ha hecho. carrera han participado con ponencias Gráfico 15.

en eventos científicos, mientras que el 


\section{Gráfico 15. Ponencias en eventos científicos}

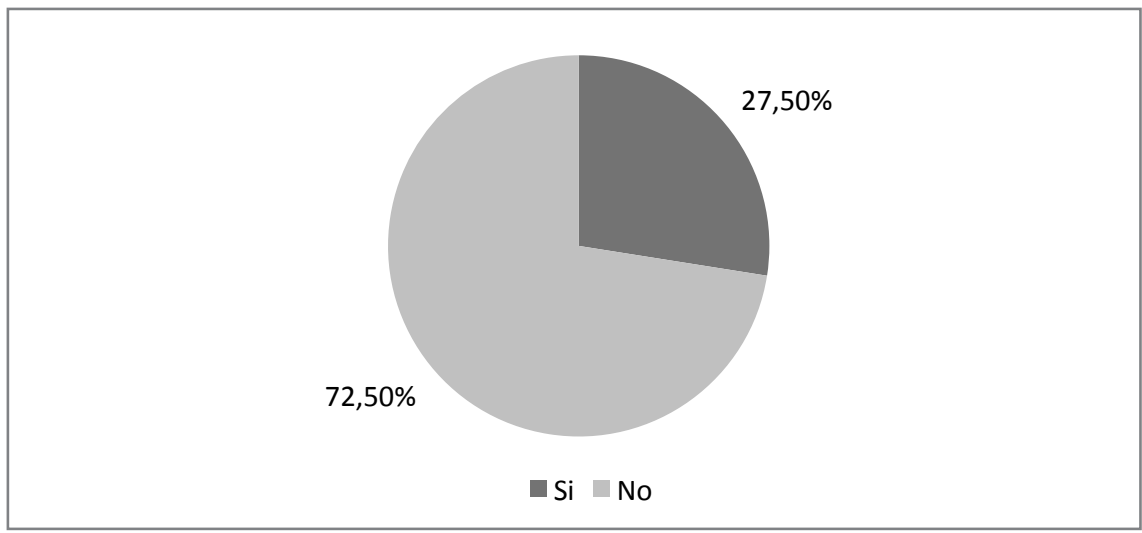

Fuente: Encuesta a docentes de la carrera de Administración de Empresas, UPS - Guayaquil

En cuanto a la participación ra ha tenido alguna participación, docente en la escritura de libros, el mientras que el $83.75 \%$ de ellos no $16.25 \%$ de los docentes de la carre- ha participado en ello. Gráfico 16.

\section{Gráfico 16. Escritura de libros}

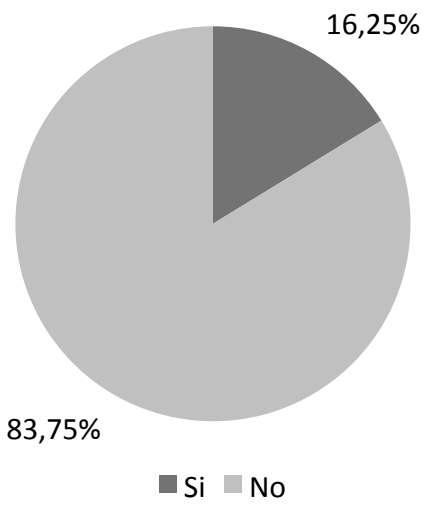

Fuente: Encuesta a docentes de la carrera de Administración de Empresas, UPS - Guayaquil

Finalmente la encuesta trató de a su producción científica, independeterminar las áreas de interés de dientemente de que ya hayan tenido los docentes de la carrera en cuanto o no publicaciones. El mayor por- 
centaje de respuestas lo obtuvo el área de administración de empresas y de derecho con un $32.2 \%$, seguido por ciencias sociales, periodismo e información con el $16.4 \%$, educación con el $15.3 \%$, servicios con el $13.6 \%$ y artes y humanidades con el
7.3\%. Al final se mencionaron otras áreas como son ingeniería, industria y construcción con el $5.1 \%$, salud y bienestar con un $4.5 \%$, ciencias naturales, matemáticas y estadística con $4 \%$ y agricultura, silvicultura, pesca y veterinaria con el $1.7 \%$.

\section{Tabla 5. Áreas de interés para la producción científica}

\begin{tabular}{|c|c|c|c|c|}
\hline & & \multirow{2}{*}{\multicolumn{2}{|c|}{ Respuestas }} & \multirow{3}{*}{$\begin{array}{c}\text { Porcentaje } \\
\text { de casos }\end{array}$} \\
\hline & & & & \\
\hline & & No & Porcentaje & \\
\hline \multirow{9}{*}{ Temática ${ }^{\mathrm{a}}$} & Educación & 27 & $15,3 \%$ & $33,8 \%$ \\
\hline & Artes y humanidades & 13 & $7,3 \%$ & $16,2 \%$ \\
\hline & $\begin{array}{l}\text { Ciencias sociales, periodismo e } \\
\text { información }\end{array}$ & 29 & $16,4 \%$ & $36,2 \%$ \\
\hline & $\begin{array}{l}\text { Administración de empresas y } \\
\text { derecho }\end{array}$ & 57 & $32,2 \%$ & $71,2 \%$ \\
\hline & $\begin{array}{l}\text { Ciencias naturales, matemáticas y } \\
\text { estadística }\end{array}$ & 7 & $4,0 \%$ & $8,8 \%$ \\
\hline & Ingeniería, industria y construcción & 9 & $5,1 \%$ & $11,2 \%$ \\
\hline & $\begin{array}{l}\text { Agricultura, sivicultura, pesca y } \\
\text { veterinaria }\end{array}$ & 3 & $1,7 \%$ & $3,8 \%$ \\
\hline & Salud y bienestar & 8 & $4,5 \%$ & $10,0 \%$ \\
\hline & Servicios & 24 & $13,6 \%$ & $30,0 \%$ \\
\hline Total & & 177 & $100,0 \%$ & $221,2 \%$ \\
\hline
\end{tabular}

Fuente: Encuesta a docentes de la carrera de Administración de Empresas, UPS - Guayaquil 


\section{Entrevistas}

Se planteó la realización de entrevistas a dos de las personas claves dentro en lo que corresponde a investigación dentro de la carrera de Administración de Empresas. El primero fue el Ing. Fabián Villacrés Beltrán, Director de la Carrera de Administración de Empresas, así mismo se entrevistó a la Dra. Raquel Ayala Carabajo, Coordinadora del Consejo de Investigación de Sede.

La entrevista se basó en cuatro preguntas abiertas de carácter exploratorio, buscando evaluar la actividad científica en la Sede y específicamente en la carrera, así como las políticas implementadas que buscan promover dicha investigación y los factores que los entrevistados consideran que limitan esta iniciativa. Finalmente se trató de determinar las líneas de investigación que se priorizan dentro de la sede y la carrera.

Entrevista a Director de la Carrera de Administración de Empresas

Sobre la evolución de la producción científica en la carrera, se afirma que en los últimos años ha existido una animación hacia los docentes en dicho sentido. Se tienen docentes que han participado en Congresos y desde ahí se pudo obtener un libro. Así mismo, ya se cuenta con docentes que están publicando en revistas indexadas, sin embargo existen muchos aspectos por mejorar.
En cuanto a las políticas para el fomento de la producción científica, se han organizado seminarios para la publicación de artículos científicos en revistas de impacto. A estos cursos asisten todos los docentes con dedicación a tiempo completo de la carrera. Se espera que en el corto y mediano plazo se tengan por lo menos unos 25 artículos publicados en revistas de impacto.

En relación a los factores que generan motivación entre los docentes, se manifiesta que al momento la motivación principal es el hecho de ya contar con sus primeros artículos en alguna revista de impacto. El docente universitario, en la actualidad, sabe que para poder tener prestigio debe tener artículos publicados lo que le da ventaja en la vida universitaria.

Sobre las líneas de investigación que se deberían priorizar en la carrera, el director afirma que se deberían concentrar en temas relacionados con: Ecología, finanzas populares, emprendimiento, innovación, manejo sostenible, marketing, responsabilidad social, sistemas de gestión de la calidad, sistemas de información geográfica, entre otras.

\section{Entrevista a Coordinadora de Conse-} jo de Investigación de Sede

Haciendo una evaluación de la producción científica en Guayaquil, se afirma que entre el 2013 y 2014 se ha dado un salto cuantitativo y cualitati- 
vo en lo que a producción científica respecta, debido a que en el 2013 se contaba con menos de una decena de productos y en el 2014 se llegó a contar con cerca de ochenta trabajos. Los involucrados han sido docentes investigadores de Guayaquil, en especial los vinculados a grupos de investigación. La calidad de la producción científica es difícil de determinar, sin embargo, la evaluación que hacen de los productos los diferentes grupos de expertos implicados y que han permitido su aceptación y publicación son un referente claro de que se está cumpliendo con los estándares de calidad exigidos. Del mismo modo, el hecho de que a partir del 2014 se tengan publicaciones en revistas indexadas en bases de datos como Scopus, es señal de que los docentes de la sede están mejorando la calidad de su producción científica.

Sobre las políticas en la sede para fomentar la producción científica, existen dos, una que se refiere a la reglamentación para la conformación de grupo de investigación y la otra referente a los incentivos económicos y el apoyo en aspectos de producción científica e investigación.

La motivación de los docentes y lo que a ella concierne, no puede clasificarse ni determinarse de forma cerrada. Según lo que se ha venido observando en el interior de los gru- pos de investigación, las motivaciones son diversas y todas legítimas y/o loables, entre las cuales destacan la difusión del conocimiento, el ascenso escalafonario, la experiencia intelectual y el perfeccionamiento docente.

La investigación en Guayaquil se desarrolla en varias líneas que son tanto del Área de Conocimiento en la Universidad Politécnica Salesiana, como la del Grupo de Investigación que la propone. No se cree que haya de privilegiar unas por encima de otras sino todo lo contrario, siguiendo siempre un principio de economía investigativa que valore las capacidades instaladas, las habilidades de los investigadores, los intereses disciplinares de las carreras, la sede y la comunidad universitaria, si hay algo que debe potenciarse en todo caso, es el trabajo interdisciplinar.

\section{Discusión}

A nivel de las universidades ecuatorianas, es posible notar en el último Ranking SIR de Scimago, la primera universidad ecuatoriana en aparecer en dicho ranking es la Universidad San Francisco de Quito en el puesto número 267 en el contexto iberoamericano y puesto 190 en el contexto latinoamericano, lo cual da muestras de la pobre participación ecuatoriana en el contexto científico internacional (Scimago Lab, 2015). 
Tabla 6. Posicionamiento de universidades ecuatorianas en el Ranking SIR Scimago 2015

\begin{tabular}{|c|c|c|c|c|}
\hline Universidad & $\begin{array}{c}\text { Posición } \\
\text { contexto } \\
\text { Iberoamericano }\end{array}$ & $\begin{array}{c}\text { Posición } \\
\text { contexto } \\
\text { Latinoamericano }\end{array}$ & $\begin{array}{l}\text { Posición } \\
\text { contexto } \\
\text { Nacional }\end{array}$ & $\begin{array}{c}\text { Total de } \\
\text { documentos } \\
\text { en revista } \\
\text { indexada en } \\
\text { Scopus }\end{array}$ \\
\hline $\begin{array}{l}\text { Universidad San Fran- } \\
\text { cisco de Quito }\end{array}$ & 267 & 190 & 1 & 422 \\
\hline $\begin{array}{l}\text { Pontificia Universidad } \\
\text { Católica del Ecuador }\end{array}$ & 304 & 222 & 2 & 319 \\
\hline $\begin{array}{l}\text { Escuela Politécnica } \\
\text { Nacional }\end{array}$ & 393 & 307 & 3 & 152 \\
\hline $\begin{array}{l}\text { Universidad Técnica } \\
\text { Particular de Loja }\end{array}$ & 399 & 313 & 4 & 143 \\
\hline $\begin{array}{l}\text { Escuela Superior Poli- } \\
\text { técnica del Litoral }\end{array}$ & 404 & 318 & 5 & 137 \\
\hline $\begin{array}{l}\text { Universidad Central del } \\
\text { Ecuador }\end{array}$ & 429 & 343 & 6 & 103 \\
\hline Universidad de Cuenca & 438 & 352 & 7 & 91 \\
\hline $\begin{array}{l}\text { Universidad Católica de } \\
\text { Santiago de Guayaquil }\end{array}$ & 439 & 353 & 8 & 90 \\
\hline $\begin{array}{l}\text { Universidad de las Fuer- } \\
\text { zas Armadas }\end{array}$ & 475 & 388 & 9 & 53 \\
\hline $\begin{array}{l}\text { Universidad Tecnológica } \\
\text { Indoamérica }\end{array}$ & 485 & 398 & 10 & 42 \\
\hline $\begin{array}{l}\text { Universidad de Especia- } \\
\text { lidades Espiritu Santo }\end{array}$ & 493 & 406 & 11 & 81 \\
\hline $\begin{array}{l}\text { Universidad de Gua- } \\
\text { yaquil }\end{array}$ & 493 & 406 & 12 & 84 \\
\hline $\begin{array}{l}\text { Universidad Politécnica } \\
\text { Salesiana }\end{array}$ & 495 & 408 & 13 & 32 \\
\hline $\begin{array}{l}\text { Universidad Nacional } \\
\text { de Loja }\end{array}$ & 498 & 411 & 14 & 29 \\
\hline $\begin{array}{l}\text { Universidad de las Amé- } \\
\text { ricas, Ecuador }\end{array}$ & 502 & 415 & 15 & 25 \\
\hline $\begin{array}{l}\text { Universidad Andina } \\
\text { Simón Bolívar, Ecuador }\end{array}$ & 505 & 418 & 16 & 22 \\
\hline
\end{tabular}

Fuente: SENESCYT, 2015 
La producción científica como tal es un factor fundamental dentro del actual sistema de evaluación y acreditación del Ecuador y por ello requiere compromiso por parte de las Instituciones de Educación Superior, así como compromiso de los docentes, para poder pasar de una línea base que en el caso de la mayoría de instituciones de educación superior es pobre a un punto en el cual haya una visualización de la institución y su producción a nivel de redes científicas relevantes.

Si bien es cierto, la producción científica en el Ecuador ha tenido incrementos en los últimos años, a pesar de ello sigue siendo pobre respecto de otros países de América Latina y el Caribe. Esto se comprueba no sólo con la cantidad de artículos publicados en revistas científicas indizadas en Scopus e ISI Web of Konowledge, sino también con la presencia de revistas indizadas en Scielo y Redalyc.

El estudio realizado entre los docentes de la Universidad Politécnica Salesiana da cuenta de algunos hallazgos interesantes, en primer lugar se evidencia que los docentes desconocen temas de normativa sobre la producción científica en la sede, conformación de grupos de investigación y los reglamentos sobre incentivos para la producción científica.

Evaluando a los docentes sobre su conocimiento respecto de herramientas básicas, se evidencia que el conocimiento tecnológico sobresale entre los docentes, mientras que en el caso de temas de conocimiento en áreas como: investigación y redacción científica, inglés y estadística, el conocimiento tiende a ser moderado. Esto debería llevar a los directivos de la sede y la carrera en particular a promover la capacitación en dicha línea para potenciar las habilidades de los docentes involucrados en procesos de investigación.

La motivación docente se encuentra entre alta y moderada y los factores que podrían hacer que dicha motivación se eleve giran en torno a tres ejes básicos como son: monetario/laboral, conocimiento y prestigio. Desde el punto de vista monetario la Universidad ya cuenta con un reglamento sobre publicaciones vigente desde el año 2014. Por el lado del conocimiento la Universidad tiene algunas opciones de cursos de redacción y producción científica que pone a disposición de sus docentes con cierta periodicidad, pero que eventualmente podría mejorar en beneficio de la calidad y cantidad de las publicaciones. En cuanto al prestigio, eso necesariamente dependerá de la tendencia creciente de las publicaciones y del direccionamiento que se le dé a las mismas, es decir, conforme aumente la calidad de las mismas, se podrán direccionar a publicaciones con mayor visibilidad a nivel internacional. 
Si aquellos que lideran los procesos de producción científica en la Universidad Politécnica Salesiana y específicamente en la carrera manejan dichos ejes, generando los incentivos adecuados, es posible que la producción científica se eleve, ya que en los actuales momentos la participación en artículos en revistas indizadas, libros y ponencias en eventos científicos es inferior al 30\% de la cantidad de docentes de la carrera.

\section{Conclusiones}

Aunque las fuentes de motivación son variadas, si existe el deseo de los docentes por realizar producción científica en el corto plazo. Las reglamentaciones sobre incentivos y grupos de investigación alientan a la generación de productos científicos, pero es necesario enfocar la capacitación docente hacia los puntos débiles detectados durante la investigación.

Una primera sugerencia es que debería existir un comité científico que canalice la producción científca de los docentes de la carrera, en el sentido de que se alinee los temas hacia las líneas de investigación de la carrera y de la institución, asimismo el comité podría hacer una labor tutorial para que los docentes se sientan respaldados y transformen sus ideas en verdaderas producciones de impacto.

Las capacitaciones en temas relacionados con la producción cientí- fica son fundamentales en este proceso, por lo cual se insta a la Dirección de la Carrera a continuar con capacitaciones no solo a nivel de redacción científica, sino también en el uso tanto de herramientas estadísticas y herramientas informáticas.

Un último aspecto, pero no por ello menos importante es que se incluya en los procesos de producción científica a los estudiantes, debido a que muchos de los proyectos de clase, como los de terminación de carrera tienen altas posibilidades de llegar a convertirse en material publicable. Esta inclusión estudiantil se está tomando muy en serio en países como México y España, teniendo excelentes resultados (Huamaní, Chávez-Solis \& Mayta-Tristán, 2008).

\section{Bibliografía}

Abadal, E. (2013). Acceso abierto a la ciencia. Barcelona: Editorial UOC.

Álvarez, L., \& Juncosa, J. (2014). La evaluación y acreditación de la educación superior ecuatoriana y la producción editorial. Universitas, 163-181.

Bozu, Z., \& Canto, P. (2009). El profesorado universitario en la sociedad del conocimiento: competencias profesionales docentes. Revista de Formación e Innovación Educativa Universitaria, 87-97.

Bruque, S. (2014). Sitio web de la Senescyt. Recuperado el 31 de Agosto de 2015 de La producción científica en Ecuador en el contexto latinoamericano: http://www.senescyt.gob.ec/adjuntos/SEMINA- 
RIO_HERRAMIENTAS_CIENTIFICAS/5\%20Sebastian \% 20 Bruque\%20Producion\%20cientifica\%20en\%20Ecuador.pdf

Consejo de Evaluación, Acreditación y Aseguramiento de la Calidad de la Educación Superior (2015). Modelo genérico de evaluación del entorno de aprendizaje de carreras presenciales y semipresenciales de las universidades y escuelas politécnicas del Ecuador. Quito: CEAACES.

Fischman, D. (2014). Motivación 360: Cómo implementarla en la vida y en la empresa. s/l: El Mercurio.

Gan, F., \& Triginé, J. (2012). Motivación. Madrid: Ediciones Diaz de Santos.

Gómez, A., Siles, G., \& Tejedor, M. (2012). Contribuyendo a la transformación social a trvés de la metodología comunicativa de investigación. Qualitative Research in Education, 36-57.

Huamaní, C., Chávez-Solis, P., \& MaytaTristán, P. (2008). Huamaní, C., Chávez-Solis, P., \& Mayta-Tristán, P. Aporte estudiantil en la publicación de artículos científicos en revistas médicas indizadas en Scielo-Perú, 1997-200. Anales de la Facultad de Medicina , 42-45.

Marina, J. (2011). Los secretos de la motivación. Barcelona: Editorial Planeta S.A.
Peña, J., Macías, N., \& Morales, F. (2011). Manual de práctica básica, motivación y emoción. México D.F.: Editorial El Manual Moderno S.A.

Peralta, M., Solis, F., \& Peralta, L. (2010). Visibilidad e impacto de la producción científica de la Universidad Central "Marta Abreu" de Las Villas durante el período 2000-2008. Revista Cubana de ACIME, 60-78.

Pirela, L., \& Prieto, L. (2006). Perfil de competencias del docente en la función de investigador y su relación con la producción intelectual. Opción, 159-177.

Presidencia de la República (12 de Octubre de 2010). Sitio web del Consejo de Educación Superior. Recuperado el 10 de Marzo de 2015, de Ley Orgánica de la Educación Superior: http://www.ces.gob.ec/descargas/ ley-organica-de-educacion-superior

Sandra, M. (2011). Revistas y producción científica de América Latina y el Caribe: su visibilidad en SciELO, RedALyC y Scopus. Revista Interamericana de Bibliotecnología, 187199.

Scimago Lab. (2015). Sitio web Scimago lab. Recuperado el 31 de Agosto de 2015, de SIR Iber 2015: http:// www.scimagoir.com/pdf/iber_new/ SIR\%20Iber\%202015\%20HE.pdf

Recepción: 02 -12-2015- Aceptación: 21- 06-2016 